\title{
Hybrid Analytical and MLS-Based NMM for the Determination of Generalized Stress Intensity Factors
}

\author{
Feng Liu, ${ }^{1}$ Hong Zheng, ${ }^{2}$ and Xiuli Du${ }^{2}$ \\ ${ }^{1}$ State Key Laboratory of Geomechanics and Geotechnical Engineering, Institute of Rock and Soil Mechanics, \\ Chinese Academy of Sciences, Wuhan 430071, China \\ ${ }^{2}$ Key Laboratory of Urban Security and Disaster Engineering, Ministry of Education, Beijing University of Technology, \\ Beijing 100124, China \\ Correspondence should be addressed to Hong Zheng; hzheng@whrsm.ac.cn
}

Received 20 September 2014; Accepted 31 December 2014

Academic Editor: Chenfeng Li

Copyright (C) 2015 Feng Liu et al. This is an open access article distributed under the Creative Commons Attribution License, which permits unrestricted use, distribution, and reproduction in any medium, provided the original work is properly cited.

The numerical manifold method (NMM) is characterized by its two cover systems, the mathematical cover and the physical cover. In the standard NMM, the mathematical cover is required to cover the whole problem domain. In this study, however, around each crack tip we specify a small domain on which the displacement is taken as the truncated Williams' displacement series. And accordingly all such small domains are not covered by the mathematical cover that only covers the rest of the problem domain. Meanwhile, the mathematical cover is constructed by designating all supports of the scattered nodes arising in the moving least squares interpolation as the mathematical patches. In this way, any physical patch contains no crack tip and can be approximated by polynomials. As a result, no blending element issue exists as in the extended finite element method and NMM. In addition to high precision, the proposed procedure is especially suitable for the situation where a crack tip is very close to other cracks, a case difficult to treat by the interaction integral procedure that is commonly used in the extraction of the stress intensity factors of mixed mode cracks.

\section{Introduction}

The extended finite element method (XFEM [1, 2]) was first introduced by Belytschko and Black and Moës et al. in 1999. The main idea of XFEM is to superpose enriched terms to the classical finite element approximation. The crack is represented independently of the mesh, allowing for simulation of the crack propagation without remeshing. It is known that the convergence rate of XFEM is influenced by the transition layer between the singular enrichment area and the rest of the domain. Some strategies for improving the convergence rate of XFEM can be found in [3-5]. Another effective approach to model crack problems is the numerical manifold method (NMM [6-14]). Besides XFEM and NMM, there are some other related methods, such as mesh-free method [15-18], cracking particles method [19], extended isogeometric analysis (XIGA [20]), and immersed particles method [21].
Usually, neither XFEM nor NMM directly estimates the stress intensity factors (SIFs). Instead, they utilize a postprocessing procedure, called the interaction integral procedure [2], to extract SIFs of a mixed mode crack. If the crack is very close to another crack, however, the interaction integral procedure cannot work very well because a local domain containing the crack tip, selected for the calculation of the $J$ integral, might be cut by the neighboring crack unless the local domain is selected very small. But if the local domain is too small, the precision of the resulting SIFs is compromised considerably.

To solve the above issue, some special techniques have been developed to conduct the crack analysis, where the SIFs are selected as the basic unknowns and can be solved directly. Liu et al. [22] enriched the approximation surrounding the crack tip with the truncated Williams' series and force the enriched DOFs to be equal. The enriched DOFs are actually 
the generalized SIFs. The numerical experiences indicate that this approach does not always reach high precision since the blending elements are still present.

Réthoré et al. [23] developed a hybrid analytical and extended finite element method, abbreviated as HAX-FEM. By HAX-FEM, the problem domain $\Omega$ is subdivided into two overlapping subdomains $\Omega_{W}$ and $\Omega_{X}$. Here, $\Omega_{W}$ is a small domain around the crack tip and, for the simplicity in presentation, $\Omega$ is assumed to contain only one crack tip. On $\Omega_{W}$ the displacement is expressed by a truncated Williams' series, while the displacement on $\Omega_{X}$ is approximated by the conventional XFEM. Due to the presence of the overlapping subdomain, the energy coupling procedure was utilized in the weak formulation so as not to commit the variational crime. In order not to avoid the overlapping subdomain, another artificial thing, Passieux et al. [24] proposed that $\Omega_{W}$ and $\Omega_{X}$ do not overlap, and the displacement continuity across the interface between $\Omega_{W}$ and $\Omega_{X}$ is enforced by the Lagrange multiplier method, which will lead to an indefinite system of linear equations. And the approximation to the Lagrange multiplier is not so easy such that the LBB condition is satisfied. To avoid the introduction of the Lagrange multiplier, recently Zhuang et al. [25] suggested that in $\Omega_{W}$ the stress is taken as the basic field variable instead of the displacement, where the weak formulation is derived from the mixed energy functional proposed by Long and Zhao [26]. This procedure is able to achieve an accurate evaluation of SIFs, but the displacement in $\Omega_{W}$ is hard to calculate accurately. The open degree of cracks is important for the situations such as the exploitation of shale gas by the hydraulic fracture technique.

In this study, the MLS-based NMM is used to evaluate directly the SIFs of mixed mode cracks. The MLS-based NMM enjoys the merits owned by the element-free Galerkin (EFG [15]) method and the NMM [6], which has been verified in the analysis of free boundary value problems such as the unconfined seepage analysis [27], and will be further confirmed by the challenging examples on crack analysis in the sequel.

\section{Elastic Crack Tip Asymptotic Fields}

The crack tip asymptotic fields, including displacement and stress fields, of a plane crack in a linear elastic brittle material with traction-free faces can be expressed in the so-called Williams' expansions [28]. If the crack lies on the negative $x$-axis and the polar coordinates centered at the crack tip are designated $r$ and $\theta$ (counterclockwise from the positive $x$-axis), Williams' series (truncated to $N$ terms) for the displacement can be written as

$$
\begin{array}{r}
u_{x}=\sum_{n=0}^{N} \frac{r^{n / 2}}{2 \mu}\left\{A _ { n } \left[\left(\kappa+\frac{n}{2}+(-1)^{n}\right) \cos \frac{n}{2} \theta\right.\right. \\
\left.-\frac{n}{2} \cos \left(\frac{n}{2}-2\right) \theta\right] \\
-B_{n}\left[\left(\kappa+\frac{n}{2}-(-1)^{n}\right) \sin \frac{n}{2} \theta\right. \\
\left.\left.-\frac{n}{2} \sin \left(\frac{n}{2}-2\right) \theta\right]\right\},
\end{array}
$$

$$
\begin{array}{r}
u_{y}=\sum_{n=0}^{N} \frac{r^{n / 2}}{2 \mu}\left\{A _ { n } \left[\left(\kappa-\frac{n}{2}-(-1)^{n}\right) \sin \frac{n}{2} \theta\right.\right. \\
\left.+\frac{n}{2} \sin \left(\frac{n}{2}-2\right) \theta\right] \\
+B_{n}\left[\left(\kappa-\frac{n}{2}+(-1)^{n}\right) \cos \frac{n}{2} \theta\right. \\
\left.\left.+\frac{n}{2} \cos \left(\frac{n}{2}-2\right) \theta\right]\right\}
\end{array}
$$

where $\mu=E /(2(1+v))$ is the shear modulus; the Kolosov constant $\kappa$ is equal to $(3-v) /(1+v)$ for plane stress and $3-4 v$ for plane strain conditions; $E$ and $v$ are Young's modulus and Poisson's ratio. The terms containing $A_{n}$ and $B_{n}$ are related to the mode I and mode II parts of deformation, respectively. Here is a brief review of some notable properties of this expansion. The rigid body translation of the crack depends on the coefficients $A_{0}$ and $B_{0}$ and the rigid body rotation with respect to the crack tip depends on $B_{2}$. The second term in the mode I expansion is often referred to as the elastic $T$ stress component. The coefficients $A_{1}$ and $B_{1}$ are related to the mode I and mode II stress intensity factors (SIFs) as

$$
A_{1}=\frac{K_{\mathrm{I}}}{\sqrt{2 \pi}}, \quad B_{1}=-\frac{K_{\mathrm{II}}}{\sqrt{2 \pi}} .
$$

Traditionally, the SIFs are important for the determination of the initiation and propagation of cracks in brittle materials. However, some recent studies show that higherorder terms of the asymptotic field are of great relevance for predicting the constraint of elastoplastic crack tip fields [29] and for interpreting the size effect of quasi-brittle materials. So evaluating the higher-order terms of Williams' expansions, called generalized stress intensity factors together with the conventional SIFs, is also necessary.

\section{Problem Formulation}

Let us consider the elastic body with a traction-free crack surface $\Gamma_{c}$ shown in Figure 1 (reproduced from [24]). The prescribed displacement $\overline{\mathbf{u}}$ is imposed on $\Gamma_{\mathcal{u}}$, while traction $t_{0}$ is acted on $\Gamma_{t}$. The domain is divided into two nonoverlapping subdomains $\Omega_{X}$ and $\Omega_{W}$ with unknown displacements $\mathbf{u}_{X}$ and $\mathbf{u}_{W}$, respectively. In domain $\Omega_{X}$, the approximation of displacement $\mathbf{u}_{X}$ is described by the MLS-based NMM, which will be discussed in the next section. In domain $\Omega_{W}$, we approximate the displacement by the truncated Williams' series given by (1).

In [24], the displacement continuity across the interface $\Gamma_{W}$ is enforced by the Lagrange multiplier method. As the number of crack tips increases, the number of Lagrange multipliers will increase rapidly. So the penalty method is adopted in this study. In fact, the results created by the two methods are very small due to the numerical excellence of 


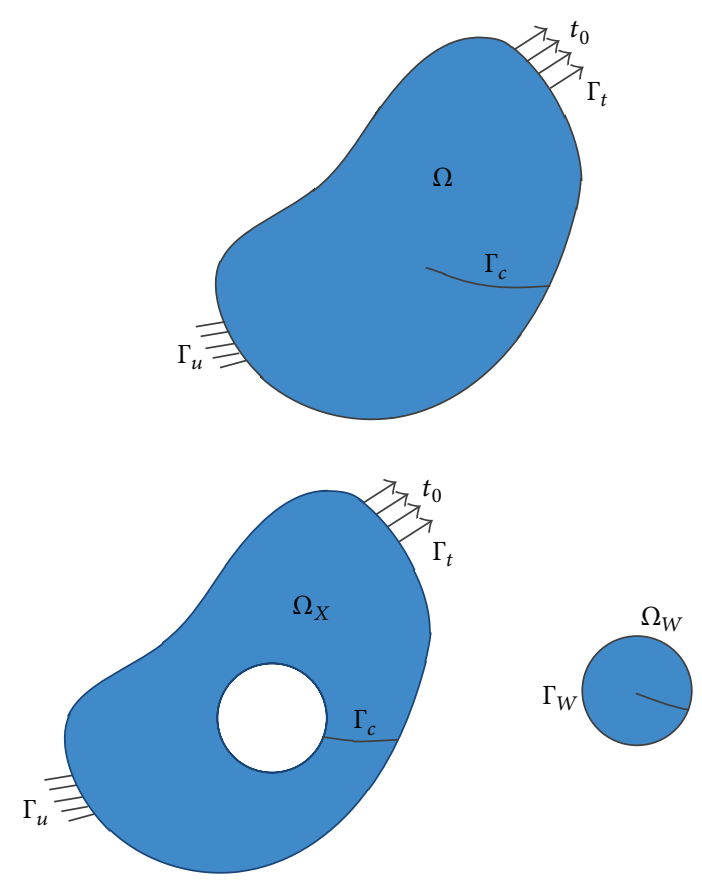

FIGURE 1: Decomposition of the domain into a standard domain and an analytical patch.

NMM; see [30], for example. In this way, we construct the conventional potential as follows:

$$
\begin{aligned}
\pi\left(\mathbf{u}_{X}, \mathbf{u}_{W}\right)= & \int_{\Omega_{X}} \frac{1}{2} \boldsymbol{\varepsilon}\left(\mathbf{u}_{X}\right)^{T} \mathbf{D} \boldsymbol{\varepsilon}\left(\mathbf{u}_{X}\right) d \Omega \\
& +\int_{\Omega_{W}} \frac{1}{2} \boldsymbol{\varepsilon}\left(\mathbf{u}_{W}\right)^{T} \mathbf{D} \boldsymbol{\varepsilon}\left(\mathbf{u}_{W}\right) d \Omega \\
& +\int_{\Gamma_{W}} \frac{1}{2} k_{p}\left(\mathbf{u}_{X}-\mathbf{u}_{W}\right)^{2} d \Gamma-\int_{\Gamma_{t}} \mathbf{u}_{X}{ }^{T} \mathbf{t}_{0} d \Gamma \\
& +\int_{\Gamma_{u}} \lambda^{T}\left(\mathbf{u}_{X}-\overline{\mathbf{u}}\right) d \Gamma,
\end{aligned}
$$

where $\boldsymbol{\varepsilon}$ is the infinitesimal strain tensor, $\mathbf{D}$ is elastic matrix, $\Gamma_{W}$ is the interface between $\Omega_{X}$ and $\Omega_{W}, k_{p}$ is the penalty factor, and $\lambda$ is the Lagrange multiplier associated with the essential boundary condition. We assume that the boundary conditions are held by $\Omega_{X}$ only since $\Omega_{W}$ is a quite small domain around the crack tip. The equation can be extended to multiple crack tips problem straightly.

3.1. Standard Subdomain. In domain $\Omega_{X}$, MLS-based NMM is used to approximate the displacement. The salient feature of NMM is the introduction of two covers, namely, the mathematical cover (MC) and the physical cover (PC). The $\mathrm{MC}$ consists of mathematical patches (MP), each of which is a rectangle or hexagon usually. The MCs do not need to match the boundary of the domain, as long as they cover the whole domain. The construction of physical patches is accomplished by cutting the mathematical patches using discontinuity lines, such as cracks and material boundaries. All the physical

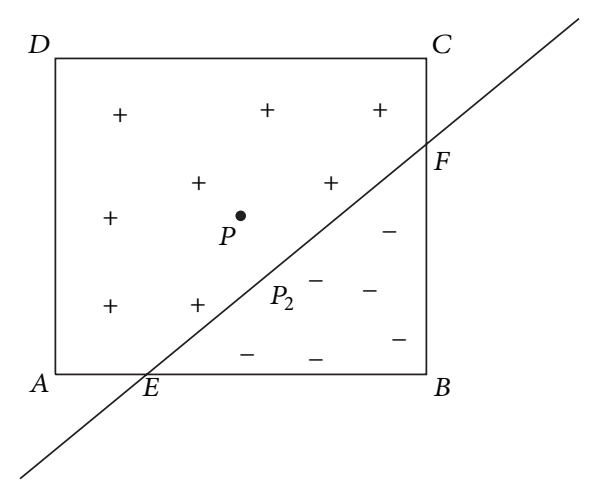

FIGURE 2: A crack cuts one mathematical patch into two physical patches.

patches form the PC covering exactly the problem domain. The main purpose to introduce two covers is to solve in a unified way the continuum and discontinuum problems. Moreover, the best approximation to the primal field variables can be guaranteed because we can always use a uniform mesh to construct the MC.

Here, we give a brief illustration on how NMM simulates the discontinuity. First, we deploy over $\Omega_{X}$ some nodes. Unlike EFG, all these nodes, called mathematical nodes, can be allowed to be outside $\Omega_{X}$, as long as their influence domains have intersection with $\Omega_{X}$. All the influence domains of these mathematical nodes constitute the MC covering $\Omega_{X}$. Let us cut the mathematical patch of node $P$ (denoted as quadrilateral $A B C D$ ) by a crack (the bold line) into 2 patches: pentagon AEFCD and triangle EBF, respectively, as shown in Figure 2. Both of the two patches are physical patches of the PC in construction. We add a new physical node, denoted by $P_{2}$, and associate $P_{2}$ with triangle EBF. At this moment, $P_{2}$ has the same coordinate as $P$. The degrees of freedom associated with the two physical nodes $P$ and $P_{2}$ are separate, reproducing strong discontinuity along the crack. So, in NMM, there is no need to introduce the Heaviside enrichment function which is widely used in XFEM. Details can be found in $[9,12]$.

For zero-order NMM, which is used in this paper, the displacement interpolation can be expressed as

$$
\mathbf{u}_{X}(x)=\sum_{i \in N_{P}} N_{i}(x) u_{i}(x)=\mathbf{N}_{X} \mathbf{U}_{X},
$$

where $N_{P}$ is the set of all physical nodes, $u_{i}$ is the degree of freedom, and $N_{i}(x)$ is the corresponding shape function with both associated with node- $i$. Here, we use the MLS shape function with linear basis and rectangular influence domain instead of the FEM shape function. The MLS interpolation is discussed in detail in $[15,31]$.

3.2. Analytical Patch. On domain $\Omega_{W}$, the displacement is described by the truncated Williams' series (1) only. So no mesh is needed theoretically. Rewrite (1) as

$$
\mathbf{u}_{W}(x)=\left[\begin{array}{l}
u_{x} \\
u_{y}
\end{array}\right]=\sum_{n=0}^{N} \frac{r^{n / 2}}{2 \mu}\left[\begin{array}{ll}
f_{1} & f_{2} \\
f_{3} & f_{4}
\end{array}\right]\left[\begin{array}{c}
A_{n} \\
B_{n}
\end{array}\right]=\mathbf{N}_{W} \mathbf{U}_{W},
$$


where $N$ is the maximum order of the truncated Williams' series; $f_{1}, f_{2}, f_{3}$, and $f_{4}$ are extracted from (1). Since the SIFs and higher-order terms are related to special $A_{n}$ and $B_{n}$ above, generalized stress intensity factors can be evaluated directly.

3.3. Matching Interface. The third term on the right of (3) stands for the matching of standard subdomain and analytical patch. No more unknowns are introduced through penalty method.

3.4. System of Equations. Though no mesh is needed for MLS interpolation, a background mesh is essential for numerical integration. In practice, a circle of radius $R_{W}=h r_{W}$ centered at the crack tip is defined first. Here, $h$ is the average element size of the mesh and $r_{W}$ is a dimensionless size controlling the region of $\Omega_{W}$. All the elements in or in intersection with the circle constitute subdomain $\Omega_{W}$, while the rest constitute subdomain $\Omega_{X}$. The outer boundary of $\Omega_{W}$, which equals the inner boundary of $\Omega_{X}$, is the common interface $\Gamma_{W}$.

Putting $\delta \pi\left(\mathbf{u}_{X}, \mathbf{u}_{W}\right)=0$ and replacing $\mathbf{u}_{X}(x)$ and $\mathbf{u}_{W}(x)$ by (4) and (5), we have the equations system as follows:

$$
\left[\begin{array}{ccc}
\mathbf{K}_{X}+\mathbf{H}_{X} & \mathbf{L}^{T} & \mathbf{G}^{T} \\
\mathbf{L} & \mathbf{K}_{W}+\mathbf{H}_{W} & 0 \\
\mathbf{G} & 0 & 0
\end{array}\right]\left[\begin{array}{l}
\mathbf{u} \\
\mathbf{a} \\
\mathbf{\Lambda}
\end{array}\right]=\left[\begin{array}{l}
\mathbf{P} \\
0 \\
\mathbf{Q}
\end{array}\right],
$$

where

$$
\begin{aligned}
& \mathbf{K}_{X}=\int_{\Omega_{X}} \mathbf{B}_{X}^{T} \mathbf{D B}_{X} d \Omega, \quad \mathbf{K}_{W}=\int_{\Omega_{W}} \mathbf{B}_{W}^{T} \mathbf{D} \mathbf{B}_{W} d \Omega, \\
& \mathbf{H}_{X}=\int_{\Gamma_{X}} \mathbf{N}_{X}^{T} \mathbf{N}_{X} d \Omega, \quad \mathbf{H}_{W}=\int_{\Gamma_{W}} \mathbf{N}_{W}^{T} \mathbf{N}_{W} d \Omega, \\
& \mathbf{L}=\int_{\Gamma_{X}} \mathbf{N}_{W}^{T} \mathbf{N}_{X} d \Omega, \quad \mathbf{G}=\int_{\Gamma_{u}} \mathbf{N}_{\lambda}^{T} \mathbf{N}_{X} d \Omega, \\
& \mathbf{P}=\int_{\Gamma_{t}} \mathbf{N}_{X}^{T} \mathbf{t}_{0} d \Omega, \quad \mathbf{Q}=\int_{\Gamma_{u}} \mathbf{N}_{\lambda}^{T} \overline{\mathbf{u}} d \Omega .
\end{aligned}
$$

$\mathbf{u}$ is the degrees of freedom in domain $\Omega_{X}$, $\mathbf{a}$ is the degrees of freedom in domain $\Omega_{W}$, and $\Lambda$ is the Lagrange multipliers associated with $\Gamma_{u}$.

To obtain $\mathbf{B}_{X}$ and $\mathbf{B}_{W}$, we should utilize the compatibility equation:

$$
\varepsilon_{i j}=\frac{1}{2}\left(\frac{\partial u_{i}}{\partial x_{j}}+\frac{\partial u_{j}}{\partial x_{i}}\right)
$$

Substituting (4) into (8) and comparing the result with equation $\boldsymbol{\varepsilon}_{X}(x)=\mathbf{B}_{X} \mathbf{U}_{X}$, matrix $\mathbf{B}_{X}$ is obtained. We can derive $\mathbf{B}_{W}$ in a similar way.

The background mesh is used for integrating the matrices in (6). For the elements cut by no discontinuity, $4 \times 4$ Gauss's points are used. For the elements cut by cracks or boundaries they are subdivided into a set of triangles and each triangle integrated by 13-point Hammer integration. For the element with a crack tip inside, a special technique [12] is used to eliminate the singularity.

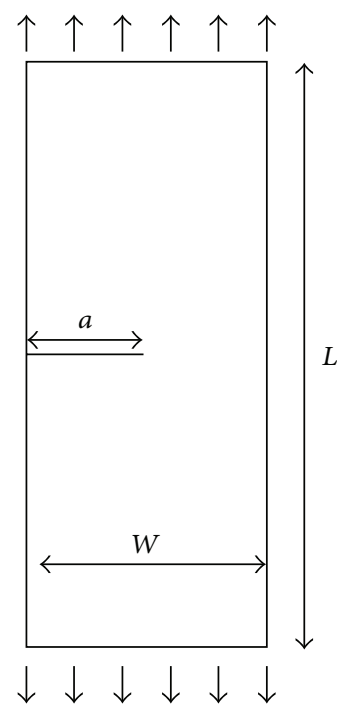

(a)

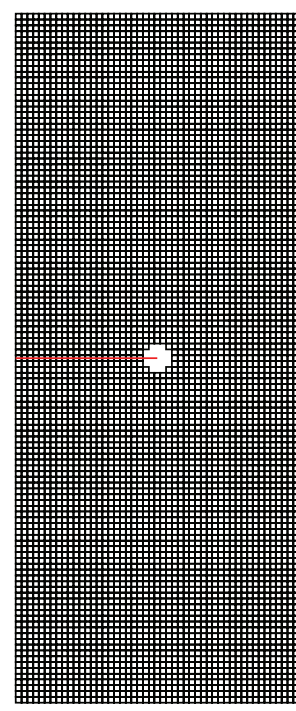

(b)
FIGURE 3: Edge-cracked plate under remote tension: (a) geometry and loading; (b) mesh and patch for $r_{W}=2$.

\section{Numerical Examples}

Several examples are studied to show the efficiency of the proposed method. The first one is an edge-cracked plate under remote tension. The choice of truncated order and the convergence are investigated. Secondly the centre-cracked plate under uniaxial tension (CCPT) and pure shearing (CCPS) are used to evaluate the generalized stress intensity factors. At last a star crack is provided to show the excellent performance of proposed method for complex cracks. In all these examples, the rectangular weight function with double node spacing is adopted.

4.1. Edge-Cracked Plate under Remote Tension. The edgecracked plate considered here is shown in Figure 3(a). To compare with [24], the parameters are as follows: $L=17 \mathrm{~mm}$, $W=7 \mathrm{~mm}, a=3.5 \mathrm{~mm}$, Poisson's ratio $v=0.3$, and Young's modulus $E=200 \mathrm{GPa}$. A state of plane stress condition is assumed.

A uniform mesh of $49 \times 119$ elements is used. The mesh, the patch (with $r_{W}=2$ ), and the crack (the red line) are plotted in Figure 3(b). The reference solution [24] of $K_{\mathrm{I}}$ for this problem is $K_{\mathrm{I} 0}=2.9637 \mathrm{MPa} \sqrt{m}$.

The normalized value of $K_{\mathrm{I}}$ is plotted as a function of the dimensionless size $r_{W}$ in Figure 4 with different orders $N$ of truncated Williams' expansion. A similar tendency is observed as in [24]. The dependence on the dimensionless size $r_{W}$ vanishes when $N$ is higher than 2 . For the same $N$, the precision decreases as the size $r_{W}$ increases, indicating that the analytical patch only influences near the crack tip. Since the influence domain of nodes in MLS is larger than that of XFEM, $r_{W}=1$ leads to a wrong solution. For general case, $r_{W}=2$ or $r_{W}=3$ is recommended and used in the following examples. 


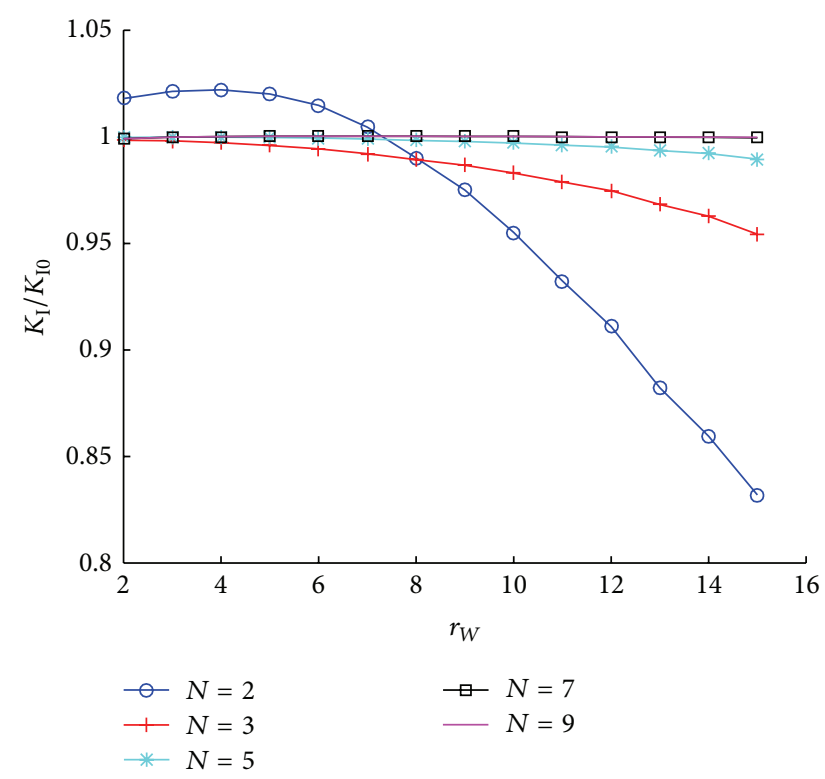

Figure 4: Normalized $K_{\mathrm{I}}$ as function of the size of the patch for different values of $N$.

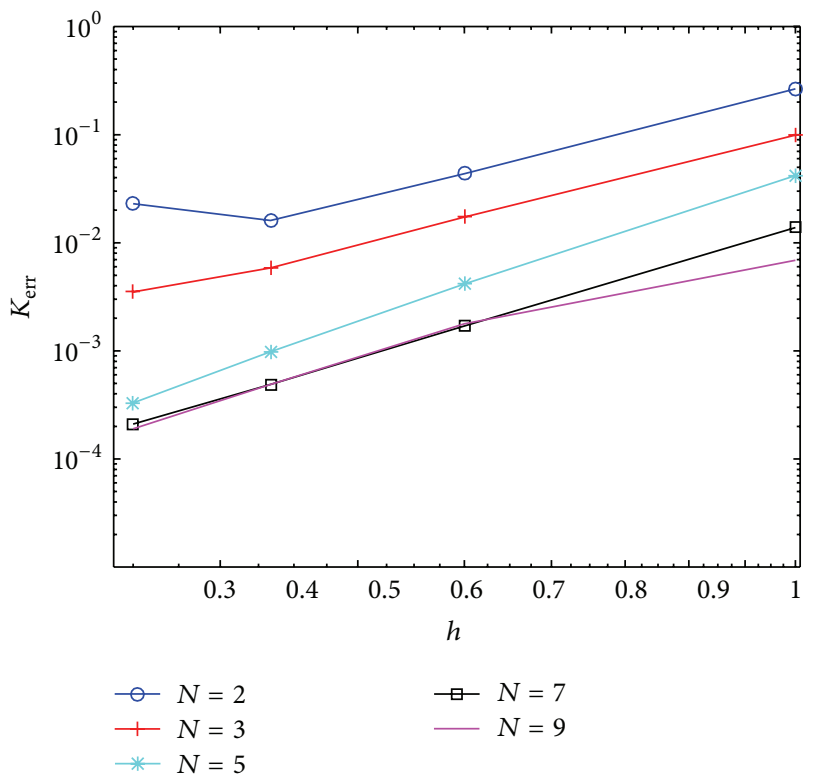

FIGURE 5: Relative error of $K_{\mathrm{I}}$ as function of node spacing for different values of $N$.

To consider the convergence of proposed method, the relative error of $K_{\mathrm{I}}$ is plotted as a function of the mesh size $h$ in Figure 5 with different orders $N$ of truncated Williams' series. The relative error of $K_{\mathrm{I}}$ is defined as

$$
K_{\text {err }}=\frac{\left|K_{\mathrm{I}}-K_{\mathrm{I} 0}\right|}{K_{\mathrm{I} 0}} .
$$

The convergence rates of relative error of $K_{\mathrm{I}}$ are around 2, larger than the value 1.25 to 1.5 in [24]. By the convergence rate we mean the average slope of $\log \left(K_{\text {err }}\right) / \log (h)$. Again we can see that when $N$ is above 2, accurate SIFs can be obtained.

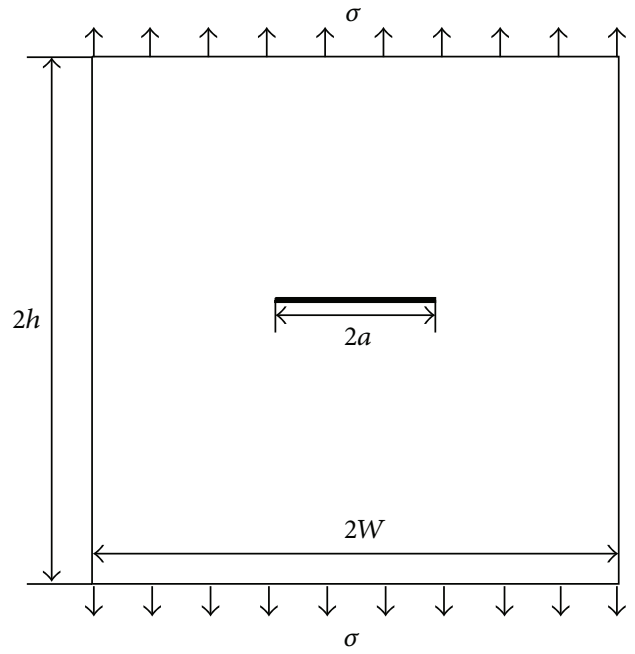

(a)

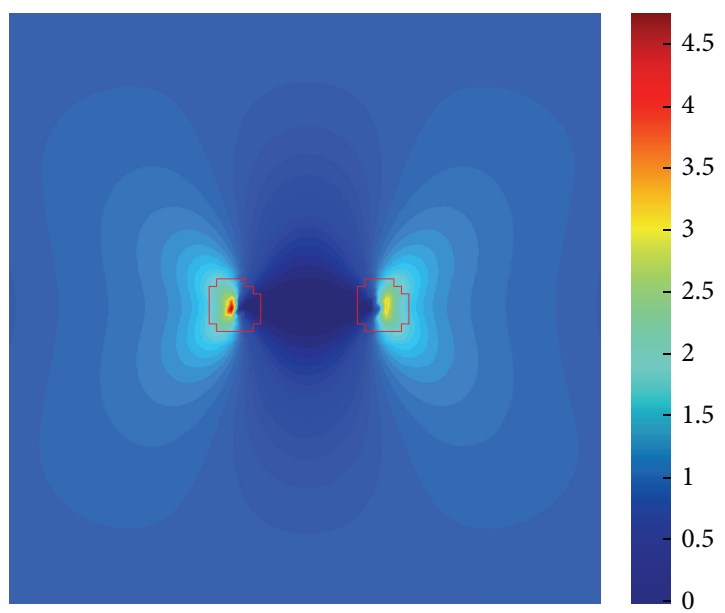

(b)

FIGURE 6: The CCPT specimen: (a) geometry and loading; (b) $\sigma_{y^{-}}$ stress nephogram for $r_{W}=3$.

For the range of penalty factor in $10^{3} \sim 10^{5} \mathrm{E}$, almost the same results are generated.

4.2. A Centre-Cracked Plate under Uniaxial Tension or Pure Shearing. The centre-cracked plate under uniaxial tension (CCPT) is selected to investigate the pure mode I crack parameters (Figure 6(a)). In order to compare the results with the reference, the following geometry and loading parameters are used: $W=h=4, \sigma=1$, and crack length $a=2$. A $80 \times 80$ mesh is used. In this case, $N=14$ together with $r_{W}=3$ are used to calculate the coefficients of Williams' expansion. The penalty factor is assumed to be $1000 \mathrm{E}$. Figure $6(\mathrm{~b})$ shows the $\sigma_{y}$-stress nephogram, where the red lines represent the common interface of $\Omega_{X}$ and $\Omega_{W}$. Table 1 shows the coefficients $A_{1}-A_{5}, B_{1}$, and $B_{3}-B_{5}$ for CCPT specimens in comparison with the results provided by other researchers $[32,33]$. It seems that the results here are in good agreement with the results of finite element overdeterministic (FEOD 
TABle 1: Coefficients $A_{1}-A_{5}, B_{1}$, and $B_{3}-B_{5}$ for CCPT specimens $(a / W=0.25)$.

\begin{tabular}{lccccccccc}
\hline & $A_{1}$ & $B_{1}$ & $A_{2}$ & $A_{3}$ & $B_{3}$ & $A_{4}$ & $B_{4}$ & $A_{5}$ & $B_{5}$ \\
\hline Present & 0.7674 & -0.0002 & -0.2761 & 0.1830 & 0.0001 & 0.0026 & 0.0001 & -0.0235 & -0.0003 \\
FEOD & 0.7679 & 0.0000 & -0.2778 & 0.1866 & 0.0000 & 0.0030 & -0.0001 & -0.0275 & -0.0000 \\
HCE & 0.7665 & - & -0.2779 & 0.1915 & - & -0.0018 & - & -0.0235 \\
BCM & 0.7680 & - & -0.2777 & 0.1866 & - & 0.0030 & - & -0.0279 & - \\
\hline
\end{tabular}

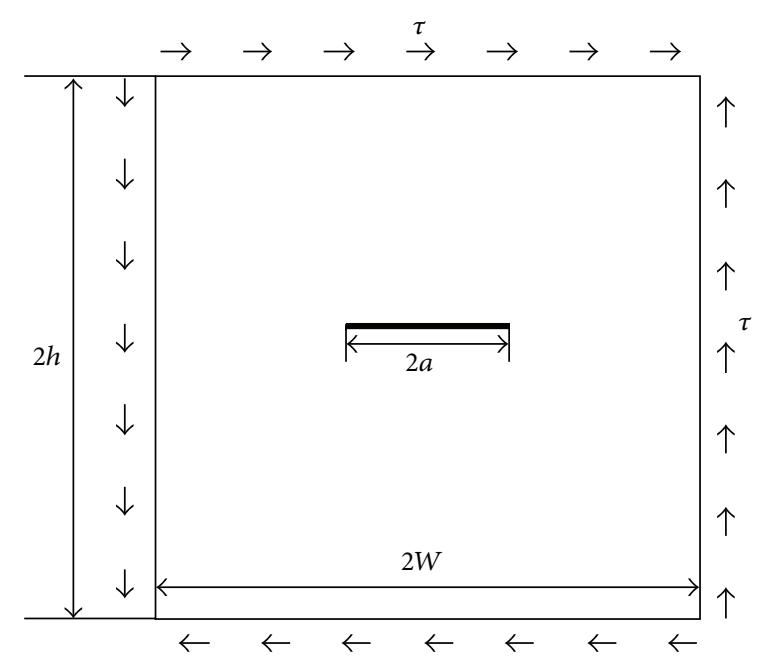

(a)

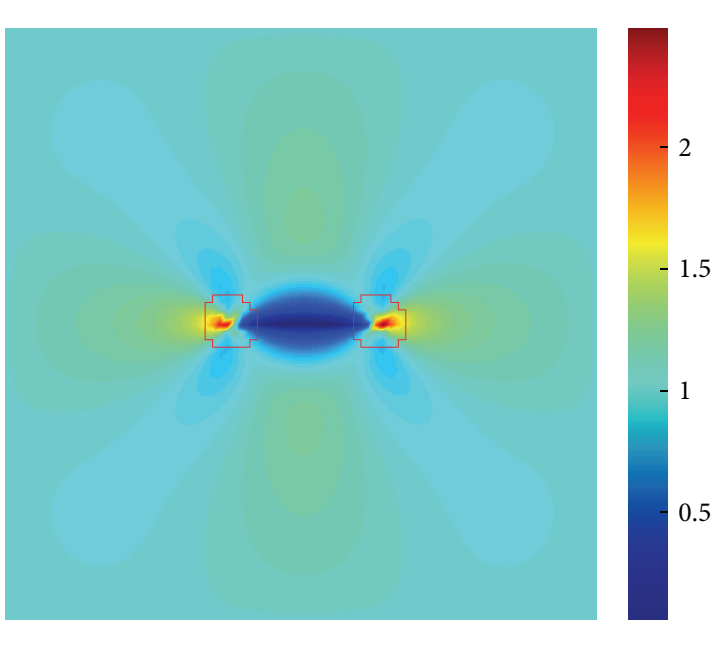

(b)

FIGURE 7: The CCPS specimen: (a) geometry and loading; (b) $\tau_{x y}$-stress nephogram for $r_{W}=3$.

[21]) method. Only $A_{1}-A_{5}$ are given for hybrid crack element (HCE) and boundary collocation method (BCM) in [32]. In fact, the results of HCE in Table 1 are calculated by half-crack model with only mode I considered. HCE considering halfcrack model with mixed mode leads to wrong results for the coefficients of higher-order parameters.

Now consider the centre-cracked plate under pure shearing (CCPS). It is a typical example of mode II. The geometrical parameters of the CCPS (Figure $7(\mathrm{a})$ ), the mesh, and material properties are the same as CCPT. Here, a uniformly distributed shear force $\tau=1$ is imposed. Figure 7(b) gives the $\tau_{x y}$-stress nephogram and again the red lines represent the interfaces. The coefficients $A_{1}-A_{5}, B_{1}$, and $B_{3}-B_{5}$ are shown in Table 2. Good agreement is observed between the present results and those of FEOD, HCE, and BCM. Again results of HCE in Table 2 are calculated by half-crack model with only mode II considered.

Both of these two examples illustrate that the present method can predict the generalized stress intensity factors precisely. No special treatment is needed throughout the whole process.

4.3. A Star-Shaped Crack in a Square Plate. At last a starshaped crack is analyzed which is often viewed as a representation of complex cracks. The star-shaped crack in a square plate subjected to biaxial tension is shown in Figure 8.
The normalized stress intensity factors at tips $A$ and $B$ are defined as

$$
F_{\mathrm{I}}^{A}=\frac{K_{\mathrm{I}}^{A}}{\sigma \sqrt{\pi a}}, \quad F_{\mathrm{I}}^{B}=\frac{K_{\mathrm{I}}^{B}}{\sigma \sqrt{\pi a}}, \quad F_{\mathrm{II}}^{B}=\frac{K_{\mathrm{II}}^{B}}{\sigma \sqrt{\pi a}} .
$$

In this example, the plate dimension is fixed to be $W=5$, and material constants are Young's modulus $E=200000$ and Poisson's ratio $v=0.3$. The biaxial tension $\sigma$ is taken to be unity. The influence of the finiteness of plate on the SIFs is also investigated. Since the mesh refinement with no limit is one of the major advantages for MLS interpolation, hierarchical meshes are used around every crack tip here. The mesh (with 4 layers of hierarchical meshes near the tip) and detailed view with analytical patches are plotted in Figure 9 with $a / W=$ 0.1 . The cracks are moved with a small distance to avoid special case caused by symmetry. Here $N=5$ and $r_{W}=3$ are selected. The computed SIF results are summarized and compared with three reference solutions, respectively, from Cheung et al. [34], Daux et al. [35], and Ma et al. [7] in Table 3. From this table, good agreement can be easily seen. This example also shows that the proposed method can implement local refinement and handle complex crack straightly.

\section{Conclusions}

A hybrid analytical and numerical manifold method is proposed to analyze crack problems. The analytical subdomain 
TABLE 2: Coefficients $A_{1}-A_{5}, B_{1}$, and $B_{3}-B_{5}$ for CCPS specimens $(a / W=0.25)$.

\begin{tabular}{|c|c|c|c|c|c|c|c|c|c|}
\hline & $A_{1}$ & $B_{1}$ & $A_{2}$ & $A_{3}$ & $B_{3}$ & $A_{4}$ & $B_{4}$ & $A_{5}$ & $B_{5}$ \\
\hline Present & -0.0002 & -0.7422 & 0.0000 & 0.0005 & -0.1848 & -0.0009 & -0.0047 & -0.0002 & 0.0264 \\
\hline FEOD & 0.0010 & -0.7403 & -0.0011 & 0.0011 & -0.1840 & -0.0003 & -0.0024 & -0.0000 & 0.0234 \\
\hline $\mathrm{HCE}$ & - & -0.7414 & - & - & -0.1835 & - & -0.0045 & - & 0.0270 \\
\hline BCM & - & -0.7422 & - & - & -0.1841 & - & -0.0034 & - & 0.0242 \\
\hline
\end{tabular}

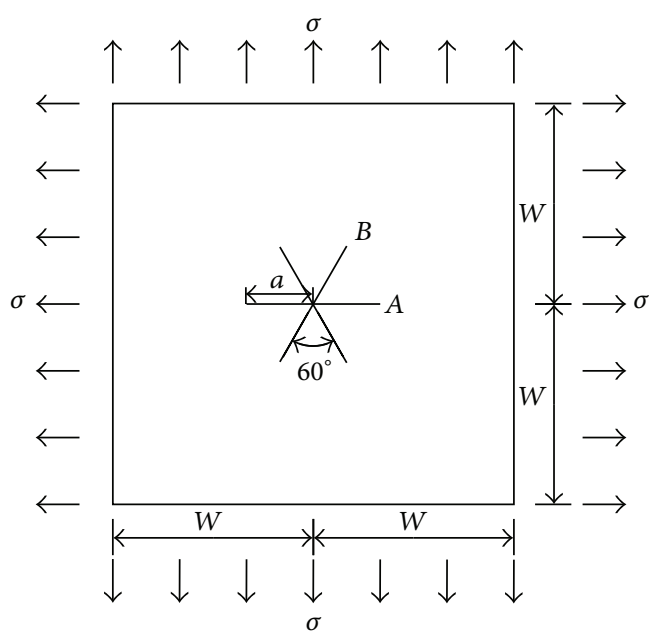

FIGURE 8: A star-shaped crack in a square plate under biaxial tension.

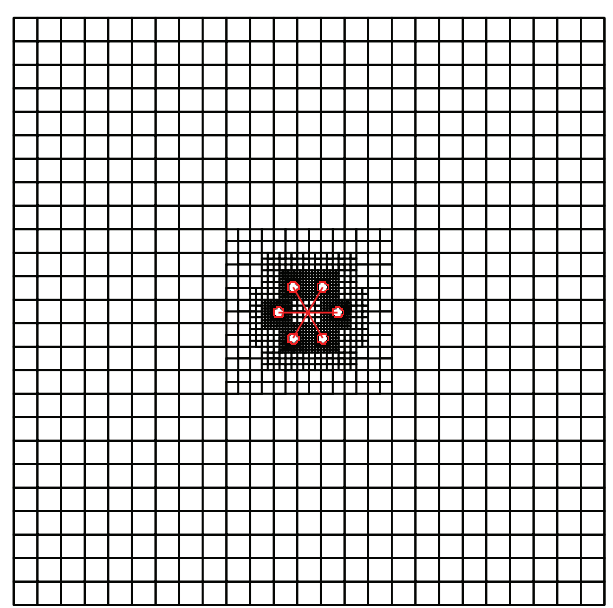

(a)

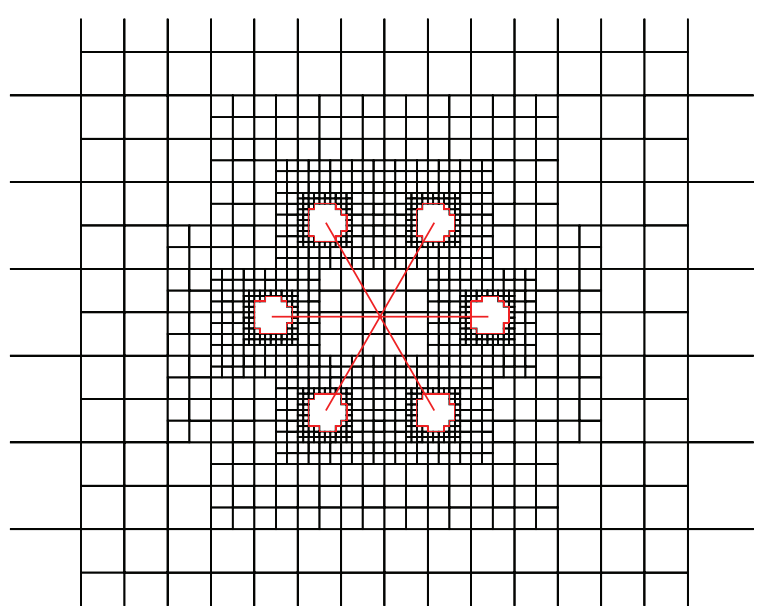

(b)

FIGURE 9: Star-shaped crack and the hierarchical meshes: (a) global view; (b) local view with analytical patches.

near the crack tip is described by the truncated Williams' expansions and the rest is modeled by the MLS-based NMM. Then, the two parts are coupled by the penalty method. The variational formulation and discrete equations are carefully discussed. The introduction of the analytical patch is to directly determine generalized stress intensity factors, while the advantage of MLS-based NMM is the simplicity in dealing with complex cracks and local refinement. The numerical experiments show that the proposed method is a promising approach for crack problems.

\section{Conflict of Interests}

The authors declare that there is no conflict of interests regarding the publication of this paper. 
TABLE 3: Normalized SIFs comparison for a star-shaped crack.

\begin{tabular}{|c|c|c|c|c|c|c|c|c|c|c|c|c|}
\hline \multirow{2}{*}{$a / W$} & \multicolumn{4}{|c|}{$F_{I}^{A}$} & \multicolumn{4}{|c|}{$F_{I}^{B}$} & \multicolumn{4}{|c|}{$F_{\mathrm{II}}^{B}$} \\
\hline & $T$ & * & $* *$ & $* * *$ & $T$ & * & $* *$ & $* * *$ & $T$ & * & ** & $* * *$ \\
\hline 0.1 & 0.7447 & 0.7408 & 0.7511 & 0.758 & 0.7463 & 0.7408 & 0.7690 & 0.767 & -0.0003 & 0.0000 & 0.0001 & 0.000 \\
\hline 0.2 & 0.7629 & 0.7570 & 0.7670 & 0.771 & 0.7649 & 0.757 & 0.7683 & 0.771 & 0.0005 & 0.0004 & 0.0005 & 0.000 \\
\hline 0.3 & 0.7913 & 0.7846 & 0.7931 & 0.789 & 0.7961 & 0.7884 & 0.7983 & 0.798 & 0.0018 & 0.0022 & 0.0021 & 0.002 \\
\hline 0.4 & 0.8319 & 0.8255 & 0.8287 & 0.821 & 0.8470 & 0.8365 & 0.8466 & 0.854 & 0.0074 & 0.0070 & 0.0080 & 0.007 \\
\hline 0.5 & 0.8887 & 0.8815 & 0.8864 & 0.887 & 0.9229 & 0.9087 & 0.9255 & 0.924 & 0.0186 & 0.0168 & 0.0184 & 0.016 \\
\hline 0.6 & 0.9712 & 0.9758 & 0.9673 & 0.971 & 1.0404 & 1.0182 & 1.0445 & 1.040 & 0.0355 & 0.0338 & 0.0364 & 0.036 \\
\hline 0.7 & 1.1035 & 1.1142 & 1.0971 & 1.107 & 1.2334 & 1.1936 & 1.2367 & 1.234 & 0.0600 & 0.0529 & 0.0593 & 0.061 \\
\hline 0.8 & 1.3398 & - & 1.3423 & 1.340 & 1.5631 & - & 1.5624 & 1.559 & 0.0847 & - & 0.0864 & 0.082 \\
\hline 0.9 & 1.8801 & - & 1.9037 & 1.930 & 2.1968 & - & 2.1927 & 2.166 & 0.0870 & - & 0.0868 & 0.089 \\
\hline
\end{tabular}

"-" means no corresponding solution.

${ }^{\star}$ Cheung et al. (1992) [34]; ${ }^{* \star D}$ Daux et al. (2000) [35]; ${ }^{* * *}$ Ma et al. (2009) [7].

\section{Acknowledgments}

This study is supported by the National Basic Research Program of China (973 Program), under Grants nos. 2011CB013505 and 2014CB047100, and the National Natural Science Foundation of China, under Grant no. 11172313.

\section{References}

[1] T. Belytschko and T. Black, "Elastic crack growth in finite elements with minimal remeshing," International Journal for Numerical Methods in Engineering, vol. 45, no. 5, pp. 601-620, 1999.

[2] N. Moës, J. Dolbow, and T. Belytschko, "A finite element method for crack growth without remeshing," International Journal for Numerical Methods in Engineering, vol. 46, no. 1, pp. 131-150, 1999.

[3] E. Béchet, H. Minnebo, N. Moës, and B. Burgardt, "Improved implementation and robustness study of the X-FEM for stress analysis around cracks," International Journal for Numerical Methods in Engineering, vol. 64, no. 8, pp. 1033-1056, 2005.

[4] P. Laborde, J. Pommier, Y. Renard, and M. Salaün, "Highorder extended finite element method for cracked domains," International Journal for Numerical Methods in Engineering, vol. 64, no. 3, pp. 354-381, 2005.

[5] E. Chahine, P. Laborde, and Y. Renard, "A non-conformal eXtended finite element approach: integral matching XFEm," Applied Numerical Mathematics, vol. 61, no. 3, pp. 322-343, 2011.

[6] G. H. Shi, "Manifold method of material analysis," DTIC Document, 1991.

[7] G. W. Ma, X. M. An, H. H. Zhang, and L. X. Li, "Modeling complex crack problems using the numerical manifold method," International Journal of Fracture, vol. 156, no. 1, pp. 21-35, 2009.

[8] G. Zhang, Y. Zhao, and X. Peng, "Simulation of toppling failure of rock slope by numerical manifold method," International Journal of Computational Methods, vol. 7, no. 1, pp. 167-189, 2010.

[9] X. An, G. Fu, and G. Ma, "A comparison between the nmm and the XFEM in discontinuity modeling," International Journal of Computational Methods, vol. 9, no. 2, Article ID 1240030, 2012.

[10] Z. Wu and L. N. Y. Wong, "Frictional crack initiation and propagation analysis using the numerical manifold method," Computers and Geotechnics, vol. 39, pp. 38-53, 2012.
[11] H. H. Zhang, "Analysis of crack interaction problem by the numerical manifold method," Advanced Materials Research, vol. 446-449, pp. 797-801, 2012.

[12] H. Zheng and D. Xu, "New strategies for some issues of numerical manifold method in simulation of crack propagation," International Journal for Numerical Methods in Engineering, vol. 97, no. 13, pp. 986-1010, 2014.

[13] G. Ma, X. An, and L. He, "The numerical manifold method: a review," International Journal of Computational Methods, vol. 7, no. 1, pp. 1-32, 2010.

[14] H. Zheng, F. Liu, and C. Li, “The MLS-based numerical manifold method with applications to crack analysis," International Journal of Fracture, vol. 190, no. 1-2, pp. 147-166, 2014.

[15] T. Belytschko, Y. Y. Lu, and L. Gu, "Element-free Galerkin methods," International Journal for Numerical Methods in Engineering, vol. 37, no. 2, pp. 229-256, 1994.

[16] G. Ventura, J. X. Xu, and T. Belytschko, "A vector level set method and new discontinuity approximations for crack growth by EFG," International Journal for Numerical Methods in Engineering, vol. 54, no. 6, pp. 923-944, 2002.

[17] X. Zhuang, C. Augarde, and S. Bordas, "Accurate fracture modelling using meshless methods, the visibility criterion and level sets: formulation and 2D modelling," International Journal for Numerical Methods in Engineering, vol. 86, no. 2, pp. 249268, 2011.

[18] M. Fleming, Y. A. Chu, B. Moran, T. Belytschko, Y. Lu, and L. Gu, "Enriched element-free Galerkin methods for crack tip fields," International Journal for Numerical Methods in Engineering, vol. 40, no. 8, pp. 1483-1504, 1997.

[19] T. Rabczuk and T. Belytschko, "Cracking particles: a simplified meshfree method for arbitrary evolving cracks," International Journal for Numerical Methods in Engineering, vol. 61, no. 13, pp. 2316-2343, 2004.

[20] S. S. Ghorashi, N. Valizadeh, and S. Mohammadi, "Extended isogeometric analysis for simulation of stationary and propagating cracks," International Journal for Numerical Methods in Engineering, vol. 89, no. 9, pp. 1069-1101, 2012.

[21] T. Rabczuk, R. Gracie, J.-H. Song, and T. Belytschko, "Immersed particle method for fluid-structure interaction," International Journal for Numerical Methods in Engineering, vol. 81, no. 1, pp. 48-71, 2010.

[22] X. Y. Liu, Q. Z. Xiao, and B. L. Karihaloo, "XFEM for direct evaluation of mixed mode SIFs in homogeneous and bi-materials," 
International Journal for Numerical Methods in Engineering, vol. 59, no. 8, pp. 1103-1118, 2004.

[23] J. Réthoré, S. Roux, and F. Hild, "Hybrid analytical and extended finite element method (HAX-FEM): a new enrichment procedure for cracked solids," International Journal for Numerical Methods in Engineering, vol. 81, no. 3, pp. 269-285, 2010.

[24] J. C. Passieux, A. Gravouil, J. Réthoré, and M. C. Baietto, "Direct estimation of generalized stress intensity factors using a threescale concurrent multigrid X-FEM," International Journal for Numerical Methods in Engineering, vol. 85, no. 13, pp. 16481666, 2011.

[25] X. Zhuang, Y. Cai, and C. Augarde, "A meshless sub-region radial point interpolation method for accurate calculation of crack tip fields," Theoretical and Applied Fracture Mechanics, vol. 69, pp. 118-125, 2014.

[26] Y. Long and Y. Zhao, "Technical note: calculation of stress intensity factors in plane problems by the sub-region mixed finite element method," Advances in Engineering Software (1978), vol. 7, no. 1, pp. 32-35, 1985.

[27] H. Zheng, F. Liu, and C. Li, "Primal mixed solution to unconfined seepage flow in porous media with numerical manifold method," Applied Mathematical Modelling, vol. 39, no. 2, pp. 794-808, 2015.

[28] M. L. Williams, "On the stress distribution at the base of a stationary crack," Journal of Applied Mechanics, vol. 24, no. 1, pp. 109-114, 1957.

[29] B. Karihaloo and Q. Xiao, "Linear and nonlinear fracture mechanics," Comprehensive Structural Integrity, vol. 2, pp. 81212, 2003.

[30] H. Zheng, Z. Liu, and X. Ge, "Numerical manifold space of Hermitian form and application to Kirchhoff's thin plate problems," International Journal for Numerical Methods in Engineering, vol. 95, no. 9, pp. 721-739, 2013.

[31] P. Lancaster and K. Salkauskas, "Surfaces generated by moving least squares methods," Mathematics of Computation, vol. 37, no. 155, pp. 141-158, 1981.

[32] Q. Z. Xiao, B. L. Karihaloo, and X. Y. Liu, "Direct determination of SIF and higher order terms of mixed mode cracks by a hybrid crack element," International Journal of Fracture, vol. 125, no. 34, pp. 207-225, 2004.

[33] M. R. Ayatollahi and M. Nejati, "An over-deterministic method for calculation of coefficients of crack tip asymptotic field from finite element analysis," Fatigue \& Fracture of Engineering Materials \& Structures, vol. 34, no. 3, pp. 159-176, 2011.

[34] Y. K. Cheung, C. W. Woo, and Y. H. Wang, "A general method for multiple crack problems in a finite plate," Computational Mechanics, vol. 10, no. 5, pp. 335-343, 1992.

[35] C. Daux, N. Moës, J. Dolbow, N. Sukumar, and T. Belytschko, "Arbitrary branched and intersecting cracks with the extended finite element method," International Journal for Numerical Methods in Engineering, vol. 48, no. 12, pp. 1741-1760, 2000. 


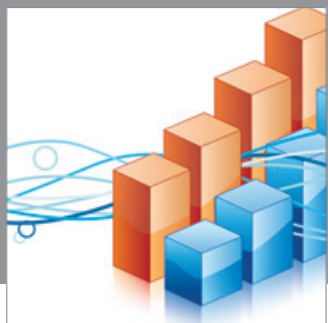

Advances in

Operations Research

mansans

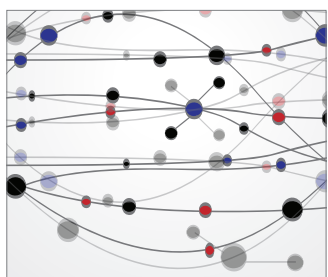

The Scientific World Journal
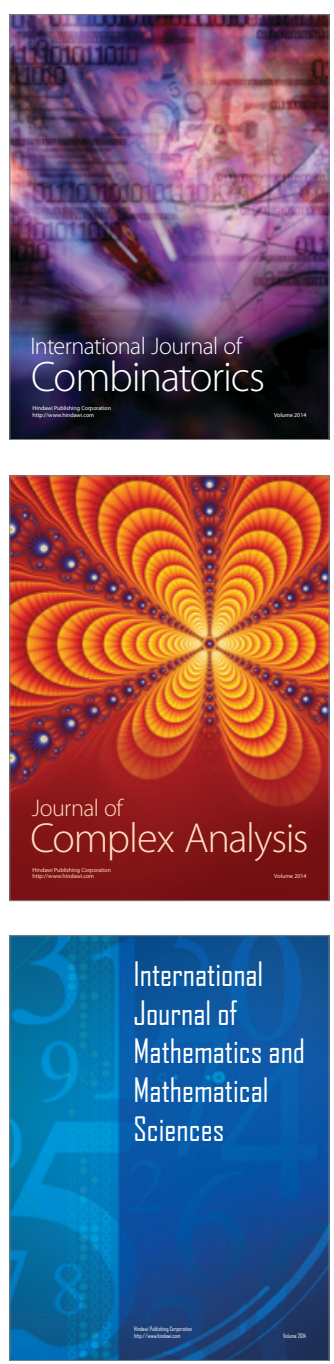
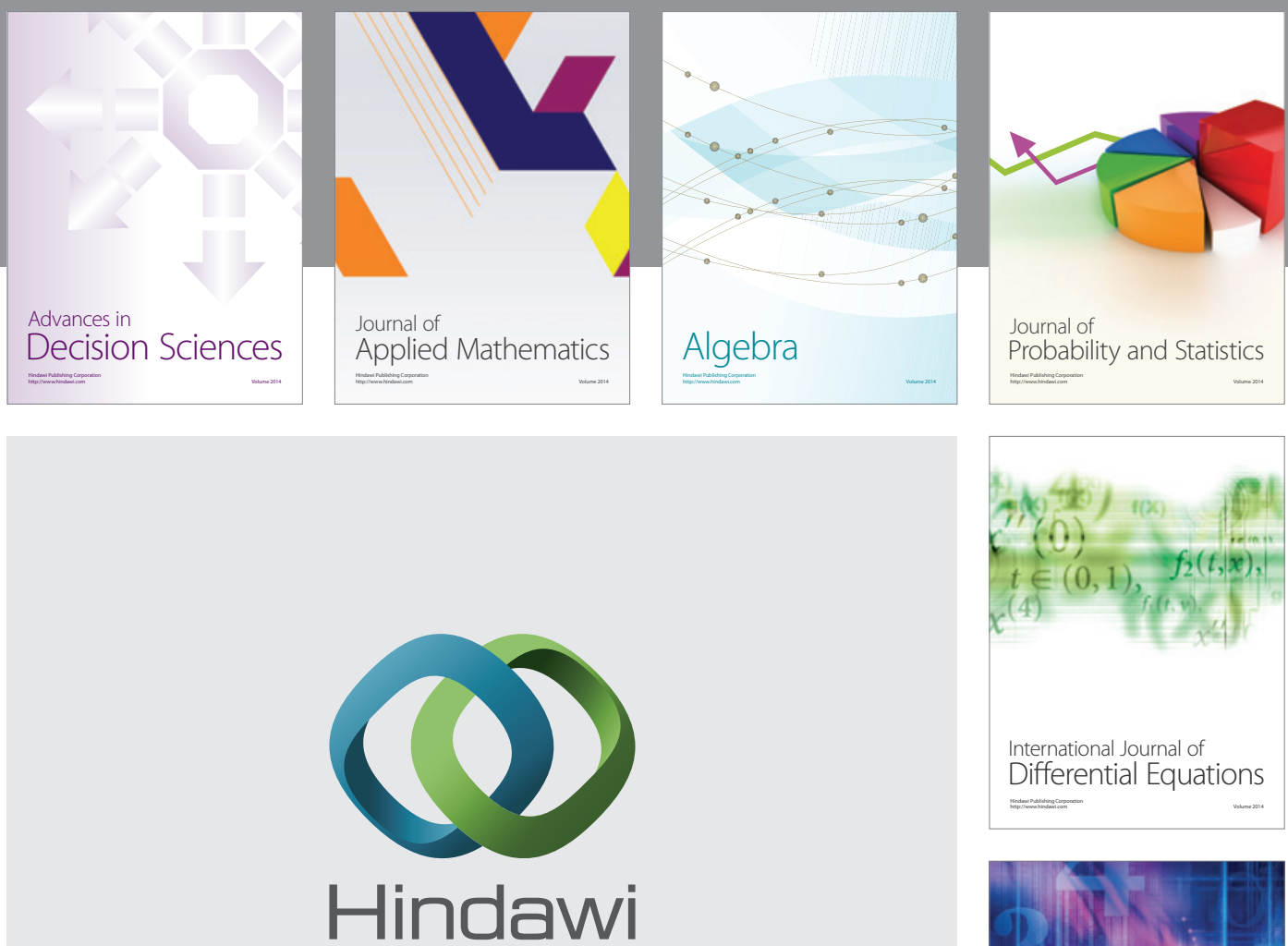

Submit your manuscripts at http://www.hindawi.com
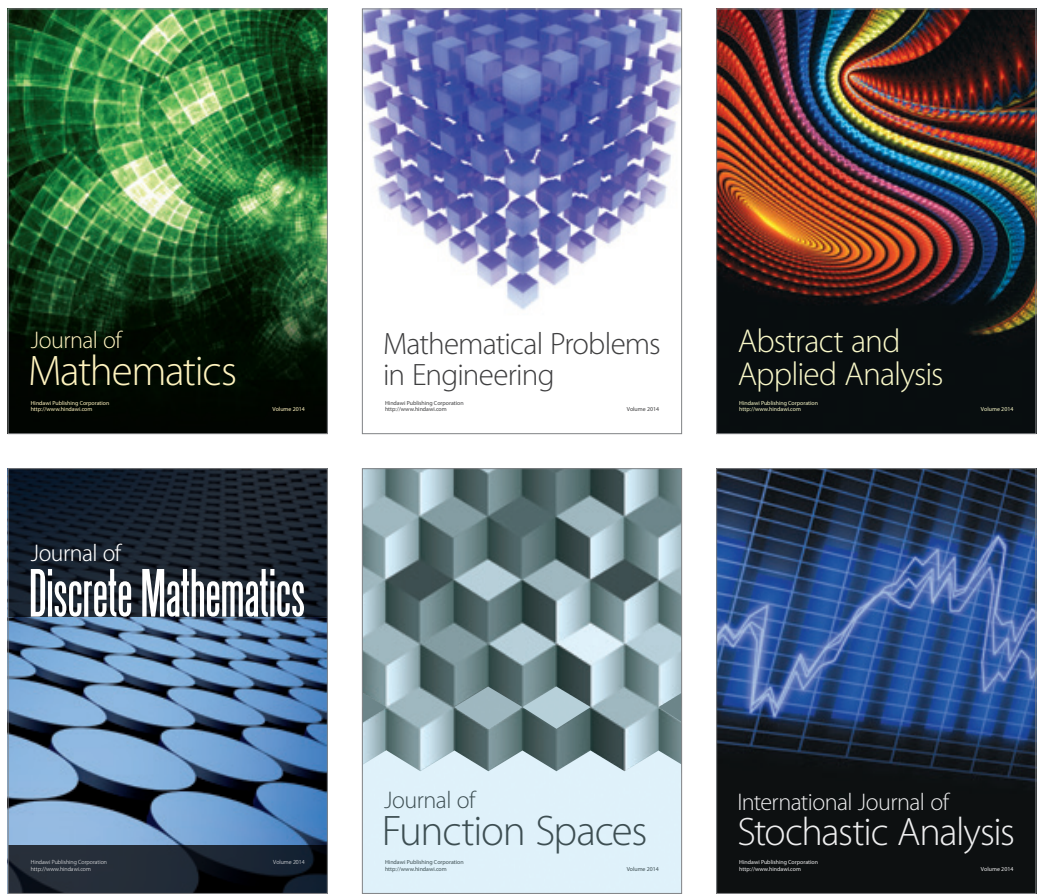

Journal of

Function Spaces

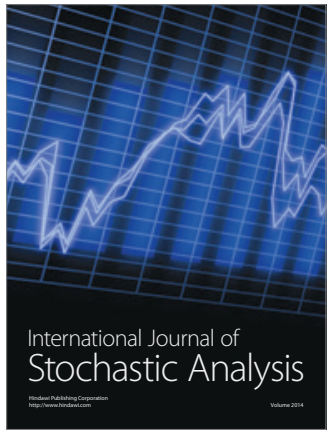

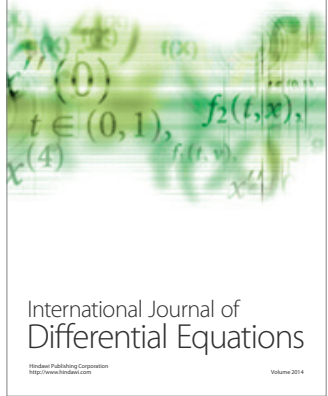
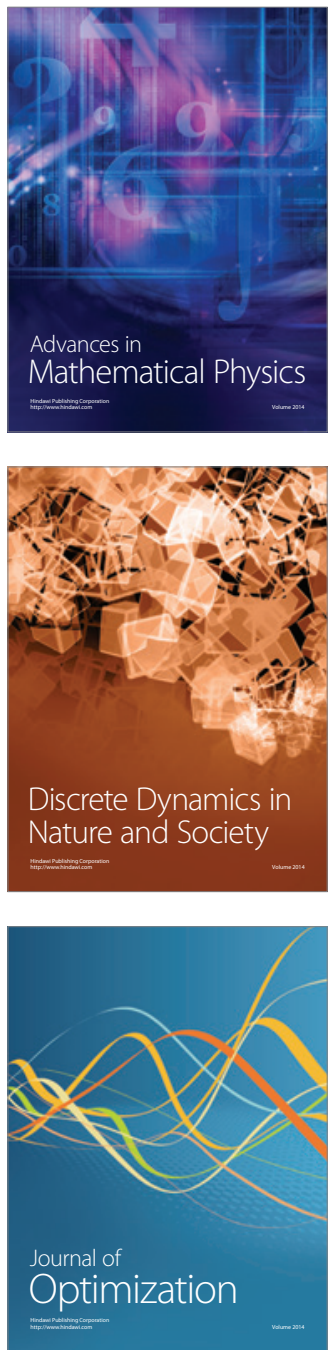